\title{
O.Louvre AF 12678: Ein koptischer Mietvertrag zwischen Muslimen?
}

\author{
Tafel XX \\ Tonio Sebastian Richter (Leipzig)
}

Abstract: Edition of an 8th century Coptic rental agreement from the Louvre collection, presumably originating from Elephantine. The lessee and perhaps the lessor too seem to be converts to Islam.

Keywords: Coptic legal documents, leases, Islamization, Elephantine

Ostrakon Louvre AF 12678 ist die dreizehnte bekannte Mieturkunde über Gebäude und Gebäudeteile in koptischer Sprache und die dritte auf einem Ostrakon aus Oberägypten. ${ }^{1}$ Sie wurde erstmals von Anne Boud'hors identifiziert und in einem Ausstellungskatalog bekannt gemacht. ${ }^{2}$ Florence Calament hat 2009 den Text erneut für einen Ausstellungskatalog beschrieben, ${ }^{3}$ und sie ist es, der ich die Publikationserlaubnis für O.Louvre AF 12678 verdanke.

Die vierlinige koptische Kursive von O.Louvre AF 12678 kann paläographisch sicher ins 8 . Jh. n.Chr. datiert werden. ${ }^{4}$ Sie ist der des bekannten thebanischen Notars Aristophanes, Sohn des Johannes, sehr ähnlich. ${ }^{5}$ Eine Datierung in frühisla-

${ }^{1} \mathrm{Zu}$ den koptischen Mieturkunden über Gebäude und Teile von Gebäuden vgl. T.S. Richter, „Koptische Mietverträge über Gebäude und Teile von Gebäuden”, JJP 32 (2002), 113-168.

${ }^{2}$ A. Boud'hors in: L'art copte en Égypte. 2000 ans de christianisme, Paris 2000, $\mathrm{n}^{\circ} 234$, p. 201 $(+\mathrm{Abb})$.

${ }^{3}$ F. Calament in: Une autre Égypte. Collections coptes du Musée du Louvre, Paris 2009, n 47 , p. 76 (+ Abb. p. 74-75).

${ }^{4}$ Zur Kursive koptischer dokumentarischer Texte vgl. A. Delattre, Papyrus coptes et grecs du monastère d'apa Apollô de Baouît conservés aux Musées royaux d'Art et d'Histoire de Bruxelles, Académie royale de Belgique, Mémoire de la Classe des Lettres 1, $3^{\mathrm{e}}$ série, Tome XLIII, no 2045, Bruxelles 2007, 127-132.

${ }^{5} \mathrm{Zu}$ diesem thebanischen Privatnotar vgl. J.A. Cromwell, „Aristophanes son of Johannes: An 8th Century Bilingual Scribe?", in: A. Papaconstantinou (ed.), The Multilingual Experience in Egypt from the Ptolemies to the Abassids. Aldershot: Ashgate 2010, 221-232; dies., „Palaeography, Scribal Practice, and Chronological Issues in Coptic Documentary Texts“, Journal of the American Research Center in Egypt 46 (2010), 1-16. Jennifer Cromwell und ich diskutierten darüber, ob O.Louvre AF 12678 von Aristophanes' Hand stammen könnte. Abgesehen von den unten genannten Gründen, O.Louvre 12678 eher in Elephantine als in Theben zu lokalisieren, sprechen gegen diese Möglichkeit einige Schreibungen vor allem griechischer Wörter, die nicht

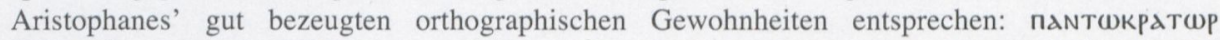

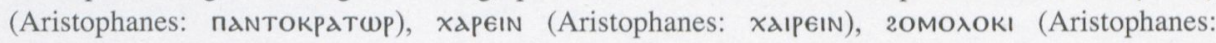


mische Zeit wird unabhängig davon durch die Ansetzung des Mietzinses in Dirham sowie dadurch erwiesen, dass Personen mit arabischen Namen agieren (s.u.).

Die Herkunft von O.Louvre AF 12678 ist unbekannt. Koptische privatrechtliche Urkunden auf Ostraka sind uns bisher ausschließlich aus dem thebanischen Raum und aus Elephantine bekannt. Sollte man zwischen diesen beiden Orten wählen, so würde man wegen der roten, glatten Scherbe und des Keramiktyps eines flachen Tellers von ursprünglich ca. $37 \mathrm{~cm}$ Durchmesser - unseren Text wohl eher in Elephantine als in Theben lokalisieren. ${ }^{6}$ Auch das Formular spricht, wenn überhaupt für einen der Orte, dann für Elephantine (s.u. zu Zeile 3-4).

Es ist insofern nicht gleichgültig, dass der Louvre neben dem British Museum und der Berliner Papyrussammlung zu den wenigen Sammlungen gehört, in welche koptische Ostraka aus Elephantine nachweislich gelangt sind. ${ }^{7}$

O.Louvre AF 12678 wird in Zeile 7 als Misth(os)is bezeichnet und ist wie alle Misthosis-Urkunden vom Mieter ausgestellt. Das Formular dieser MisthosisUrkunde fällt allerdings kürzer als gewöhnlich aus. Außer den Geschäftsparteien und dem Ausstellungsdatum werden in der Urkunde lediglich das Mietobjekt (ein „Zimmer in deinem Haus“ ohne weitere Lagebeschreibung), die Höhe des Mietzinses (ein Dirham per annum) und die Mietdauer von einem Jahr ,ab dem heutigen Tag" festgehalten.

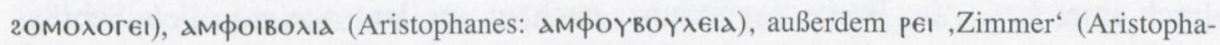
nes: $\mathrm{pl}$ ).

${ }^{6}$ Diesen Eindruck vermitteln nicht nur die wenigen publizierten koptischen Ostraka aus Elephantine (siehe Fn. 6), sondern auch die vielen unpublizierten, zu denen mir freundlicherweise mein Leipziger Kollege Dr. Dietrich Raue mitteilt: „Auf Elephantine werden für den alltäglichen Schriftverkehr durch Ostraka neben einfacheren Amphorenscherben regulär und in großer Zahl Fragmente der hochwertigen Mergelwaren der spätantik-frühislamischen Zeit, von denen auch das Bruchstück im Louvre stammt, verwendet.“

7 S. Bacot \& Ch. Heurtel, „Ostraca coptes d'Élephantine au musée du Louvre“, in: N. Bosson (Hrsg.), Études coptes VII. Neuvième journée d'études, Montpellier 3-4 juin 1999, Cahiers de la bibliothèque copte 12, Paris/Louvain/Sterling/Virginia: Peeters 2000, 17-45 (reed. SB Kopt. III, 1288-1291, 1441, 1384-1393, 1657, 1665). Weitere edierte koptische Elephantine-Ostraka: O.Brit. Mus. Copt. I, pl. 19/4, 22/3 (= P.Rain. Unterricht kopt. 100); 44/4; 47/1; 51/4; 83/4; 88/5; 90/4; Add. 19-21 (vgl. A. Delattre, ,Noms rares et noms fantômes dans trois ostraca grecs d'Éléphantine“, CdE 85 (2010), 369-373), R. Engelbach, ,A Coptic Ostrakon mentioning lèb (Elephantine)“, ASAE 38 (1938) 47-51; F. Hintze, ,Berliner koptische Ostraka aus Elephantine“, ZÄS 104 (1977), 97-112 (reed. SB Kopt. I, 024-035, 235; K.A. Worp, „Das Berliner Ostrakon P. 14735: Koptisch oder Griechisch?", APF 36, 1990, 74-77), T. Markiewicz, „Three Coptic Ostraca from Warsaw Private Collections“, JJP 28 (1998) 95-98, n 2 \& 3 (reed. SB Kopt. III 1648, 1669); O.ÄMUL inv. 7217 in S. Hodak, T.S. Richter \& F. Steinmann, Coptica. Koptische Ostraka und Papyri, koptische und griechische Grabstelen aus Ägypten und Nubien, spätantike Bauplastik, Textilien und Keramik, Berlin 2012, Kat.-Nr. 7. 


\section{Text und Kommentar}

Maße:

Beschreibung:

Schrift:

Herkunft:

Datierung:

Aussteller/Mieter:

Adressat/Vermieter:

Mietobjekt:

Mietdauer:

Mietzins:
H. $9,3 \mathrm{~cm} \times$ B. $20,4 \mathrm{~cm}$

Rote, glatte, dünne Scherbe: Rand eines Tellers. Unten links Ausbruch

Vierlinige Kursive mit Ligaturen

Unbekannt, evtl. aus Elephantine (vgl. Einleitung)

8. Jh.

Sulaiman, Sohn des Georg

Jannia, Tochter des Meisters Athanasi

Ein Zimmer im Haus der Vermieterin

1 Jahr ab dem Ausstellungstag, dem 24. Tybi des 10. Ind.-Jahres

1 Dirham

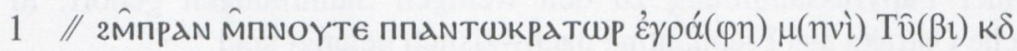

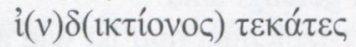

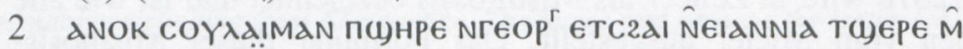

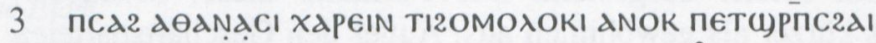

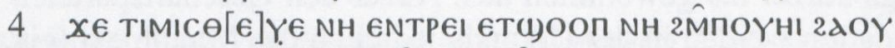

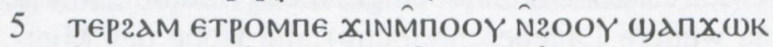

6 NNTPOMIE NNATAdAY N̂()dXE N̂NAMQOIBO

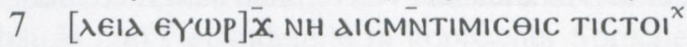

(spatium)

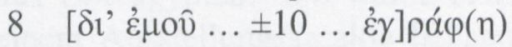

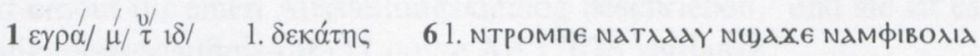

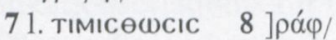

1 Der Textbeginn von O.Louvre AF 12678 ist statt durch ein Staurogramm durch einen bzw., der Breite des Striches nach zu urteilen, zwei parallele Schrägstriche markiert, deren Funktion im epistolaren Dekorum darin bestand, eine Anpassung der Symbolik an die Empfindlichkeiten nicht-christlicher, namentlich muslimischer Kontrahenten zu bewirken. ${ }^{8}$

Die Invokatiosformel ist, wie in vielen späteren koptischen Dokumenten, rein monotheistisch. ${ }^{9}$ Unter dem Gesichtspunkt, dass Aussteller und Adressatin der Urkunde vermutlich Konvertiten zum Islam sind (siehe zu Z. 2-3), erscheint es immerhin bemerkenswert, dass diese Invokation sich nicht auf die bloße Gottesbezeichnung beschränkt, sondern das Epitheton „Allherrscher“ einschließt, das freilich in Epitheta Allahs wie al- 'Azīz (z.B. Sure $3_{9}, 4_{158}$ u.ö.), al-Qādir (z.B. Sure $46_{33}$ ) oder Mālik al-mulk (z.B. Sure $3_{26}$ ) sachliche Entsprechungen findet.

8 Vgl. T.S. Richter, ,Spätkoptische Rechtsurkunden neu bearbeitet (III): P.Lond. Copt. I 487, Arabische Pacht in koptischem Gewand“, JJP 33 (2003), Appendix: „Das Symbol // (u.ä.) anstelle des Kreuzes am Anfang koptischer Texte“, 223-230 mit Hinweisen auf ältere Literatur.

9 T.S. Richter \& G. Schmelz, ,Der spätkoptische Arbeitsvertrag P.Heid. kopt. inv. 451 “, JJP 40 (2010), 185-203, Anhang: „Die Invokationen in den koptischen Urkunden“, 201-203. 
Das Datum der Ausstellung ist zugleich der Mietbeginn (siehe Z. 5-6). Nach den Daten zu urteilen, die in den koptischen Mieturkunden ent- bzw. erhalten sind, begannen die Mietverhältnisse oftmals nicht zum Ersten des Monats (s. die Tabelle). In der thebanischen Mieturkunde O.Crum Ad. 15 läuft der Mietvertrag z.B. ebenfalls ab dem 24. eines Monats.

\begin{tabular}{|l|l|l|}
\hline Referenz & Monat & Tag \\
\hline BKU III 426 & Thot & Tag 1 \\
\hline CPR IV 113 & $\ldots$ & Tag 1(?) \\
\hline CPR IV 114 & Pachons & Tag 20 \\
\hline P.Lond. Copt.I 1017+ & Paone & Tag 20 \\
\hline P.Lond. Copt.I 1018 & $\ldots$ & Tag 4 \\
\hline P.Lond. Copt. I 1023 & Hathor & Tag [1]2 oder [2]2 \\
\hline P.Ryl. Copt. 171 & Paone & Tag 4 \\
\hline P.Ryl. Copt. 172 & Paone & Tag 4 \\
\hline O.Crum Ad. 15 & Mechir & Tag 24 \\
\hline O.Brit. Mus. Copt.I $73 / 2$ & Pachons & Tag 11 \\
\hline
\end{tabular}

2-3 Die Intitulatio des Ausstellers und die Adresse des Empfängers in O.Louvre AF 12678 sind gemäß dem Formular oberägyptischer Briefe ${ }^{10}$ mit der Cleft senctence $A \in \operatorname{Tc} 2 \alpha I \mathrm{~N}-B \times \boldsymbol{x}(1)$ peIN , $A$ ist es, der an $B$ schreibt, zum Gruße!“ formuliert, wie es auch in den beiden anderen bekannten oberägyptischen Mieturkunden O.Crum Ad. 15 und O.Brit. Mus. Copt. I 73/2 aus dem thebanischen Raum bzw. Hermonthis der Fall ist. Die Vermieterseite in O.Louvre AF 12678 wird von einer Frau repräsentiert - eine in den koptischen wie auch in den viel zahlreicheren griechischen Mieturkunden auffallend häufige Konstellation, ${ }^{11}$ für die meines Wissens noch keine Erklärung gefunden ist. Der Frauenname Jannia der Adressatin/Vermieterin ist kein typisch koptischer und auch kein arabischer Name, im übrigen scheint er nur selten belegt zu sein. ${ }^{12}$ Der Name des Ausstel-

10 A. Boudhors \& Ch. Heurtel, Les Ostraca Coptes de la TT29. Autour du moine Frangé. Études d'archéologie thébaine 3. Bruxelles 2010, vol. II, 66-68.

${ }^{11}$ Schon A. Berger, ,Wohnungsmiete und Verwandtes in den gräko-ägyptischen Papyri“, Zeitschrift für vergleichende Rechtswissenschaft 29 (1913), 321-415, schreibt (355): „Es ist erstaunlich, wie oft in den Mietverträgen Frauen, sowohl als Vermieterinnen als auch als Mieterinnen ...,

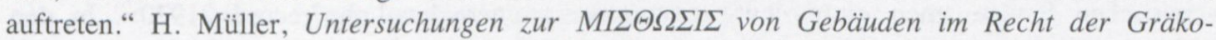
Ägyptischen Papyri. Erlanger Juristische Abhandlungen 33. Köln/Berlin/Bonn/München 1985, 102-109, berechnete den Frauen-Anteil in Mieturkunden der Prinzipatszeit auf ein Sechstel, für die byzantinischen Texte sogar auf ein Drittel. Für die koptischen Texte vgl. Richter, Koptische Mietverträge (Fn. 1), 139.

${ }^{12} \mathrm{Zu}$ diesem Frauennamen vgl. Hans Förster, ,Zahlungsanweisung zugunsten von Jannia“, in: F.A.J. Hoogendijk/B.P. Muhs (Hrsg.), Sixty-Five Papyrological Texts Presented to Klaas A. Worp on the Occasion of his 65th Birthday, Leiden 2008, Pap.Lugd. Bat. 33, 351-359, speziell 356f. Die frühesten Belege sind P.Oxy. 2480 und P.Sorb. II, 21,37; 27 (passim); 28,4, 92,12 aus dem 6. Jh. An koptischen Belegen sind P.Vindob. K 4737 (6.-7. Jh.), P.Rain. Unterricht kopt. 105 (8.-9. Jh.) und die nubischen Stelen T. Mina, Inscriptions coptes et grecques de Nubie, $\mathrm{n}^{\circ} 117$ und $\mathrm{n}^{\circ} 225$ 
lers/Mieters coYdaıman ist zweifelsfrei arabisch: Die arabische Form des Namens Salomon ist in koptischen Texten seit dem 8 . Jh. recht häufig bezeugt. ${ }^{13}$ Beide Kontrahenten, Iannia und Sulaiman, haben Väter mit dezidiert christlichen Namen (Georg, Athanasi), und sie bedienen sich der koptischen Sprache. So ist es alles in allem wahrscheinlich, dass wir es hier mit mindestens einem, wenn nicht zwei Konvertiten zum Islam zu tun haben haben - ein für Oberägypten im 8. Jh. zweifellos bemerkenswerter Befund!

3-4 Die Mieterklärung von O.Louvre AF 12678 ті2омодок। ... xє єІмІ$\mathrm{c} \Theta[\epsilon]$ Y $€ \mathrm{NH}$, ich bekenne, dass ich von dir miete“ ist jener der ausführlich stilisierten koptischen Misthosis-Urkunden aus Aschmunein ähnlicher als jener der beiden in puncto Herkunft, Schreibmaterial und Kürze dem Louvre-Ostrakon näherstehenden oberägyptischen Mieturkunden: O.Crum Ad. 15 benutzt dafür den Ausdruck Tı ,geben“, der allein durch die Gegenleistung von (yкגP „Mietzins“ als ein „Geben zur Miete“ konnotiert ist; O.Brit. Mus. Copt. I 73/2 gebraucht das alte ägyptische Rechtswort P-Cd2NG Nd=,,jemandem eine Miet-/Pacht-Urkunde machen". ${ }^{14}$ Zwei oberägyptische Misthosis-Urkunden, welche Landpacht zum Gegenstand haben, BKU I 82 und O.Crum VC 33, bedienen sich ebenfalls nicht des

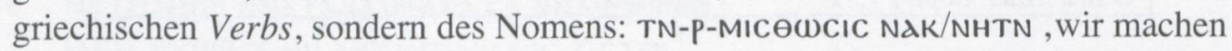
dir/euch eine Misthosis-Urkunde'. Dagegen formuliert das Fragment einer Mietoder Pachturkunde aus Elephantine O.Brit. Mus. Copt. I 51/4 wie die MisthosisTexte aus Aschmunein TI-MiceoY N€ "Ich miete/pachte von Dir“, und dies ist auch die Formel, die O.Louvre AF 12678 bietet. Im Unterschied zu den bisher bekannten Belegen dieser Formel, in denen eine vom griechischen Infinitiv Präsens Aktiv derivierte Verbform miceoy (+ Dativ) in der dem griechischen Medium entsprechenden Bedeutung ,anmieten, anpachten (von)“ gebraucht wird, ${ }^{15}$ scheint O.Louvre AF 12678, falls unsere Lesung und Rekonstruktion stimmen, in derselben Bedeutung eine hier erstmals belegte Verbform micøєү€ (+ Dativ) zu verwenden.

4-5 Die Höhe des Mietzinses und seine Spezifizierung im arabischen Silberkurrant, dem Dirham, entspricht der thebanischen Mieturkunde O.Crum Ad. 15, wo für einen Dirham (weniger $2 \mathrm{Habba}$ ) p.a. allerdings nicht nur ein Zimmer, sondern ein Haus vermietet wird. Der Terminus ()6op / ()kap ,Mietzins ‘ ${ }^{16}$ der in

(jeweils 9. Jh.) zu nennen. Die Datenbank Trismegistos People identifiziert 'Iavveía/ıannid als semitischen Frauennamen <http://www.trismegistos.org/nam/detail.php?record=21970>. In den arabischen Papyri findet er sich jedoch nicht, wie mein Leipziger Kollege Boris Liebrenz freundlicherweise für mich nachprüfte.

${ }^{13}$ M. Legendre, ,Répertoire des anthroponymes arabes trouvés dans les documents coptes," in: A. Boud'hors, A. Delattre, C. Louis \& T.S. Richter (Hsgg.), Coptica Argentoratensia. Conférences et documents de la $3 e$ université d'été internationale en papyrologie copte (Strasbourg, 18-25 juillet 2010). Cahiers de la Bibliothèque Copte. Louvain: Peeters 2013, s.v.

14 T.S. Richter, „Alte Isoglossen im Rechtswortschatz koptischer Urkunden,“ Lingua Agyptia 10 (2002), 394f.

15 Vgl. Richter, Koptische Mietverträge (Fn. 1), 117-119.

${ }^{16}$ Vgl. Richter, Koptische Mietverträge (Fn. 1), 116-117. 
allen anderen koptischen Mieturkunden gebraucht ist, findet sich in O.Louvre AF 12678 nicht.

5-6 Die vereinbarte Mietdauer von einem Jahr ist eine mehrfach belegte Frist, die z.B. auch in der thebanischen Mietvereinbarung O.Crum Ad. 15 vereinbart wird. ${ }^{17}$ Der Beginn der Mietdauer ,von heute an“, d.h. die Urkundenausstellung am ersten Tag der Mietfrist, ist ebenfalls gut belegt. ${ }^{18}$

7 Die Ergänzung des Zeilenanfangs ist für die Größe der Lücke etwas kurz, doch angesichts des unmittelbar nachgestellten Dativs NH "für dich“ kommt nur die kurze Variante $\epsilon\} \omega p x$, zur Sicherheit“, nicht eine der längeren Varianten (6-

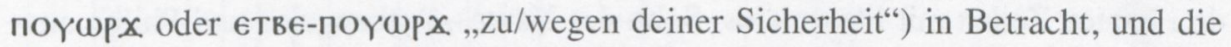
Präposition єтвє- ist in dieser Formel nur zusammen mit dem Possessivartikel, nicht mit dem unbestimmten Artikel belegt.

8 Der dem Schriftbild nach zu urteilen professionelle Notar, ${ }^{19}$ der die kurze Misthosis-Urkunde aufgesetzt hat und hier mit seinem Fertigungsvermerk beendet, bleibt uns wegen des Ausbruchs am unteren linken Rand des Ostrakon leider unbekannt.

\section{Übersetzung}

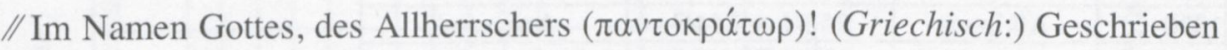
am 24. des Monats Tybi des zehnten Indiktionsjahrs.

(Koptisch:) Ich, Sulaiman, der Sohn des Georg, bin es, der an Jannia, die Tochter

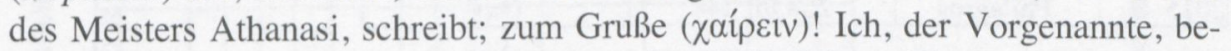

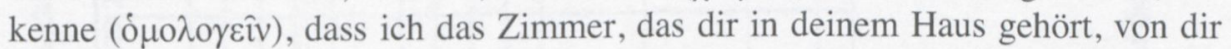
miete $\left({ }^{*} \mu 1 \sigma \theta \varepsilon v ́ \varepsilon v v\right)$ für einen Dirham pro Jahr vom heutigen Tag an bis zur Vollendung des Jahres, ohne ein Wort des Einwandes ( $\alpha \mu \varphi \imath \beta 0 \lambda i ́ \alpha)$. Zur Sicherheit für dich habe ich diese Misthosis ( $\mu$ í $\theta \omega \sigma \mathrm{c})$ ) ausgestellt, (und) ich stimme zu ( $\sigma \tau \mathrm{\tau} \chi \varepsilon \hat{\mathrm{i} v})$.

(Griechisch:) [Durch mich, ..., , geschrieben.

${ }^{17}$ Richter, Koptische Mietverträge (Fn. 1), 142-143. In den griechischen Mieturkunden byzan-

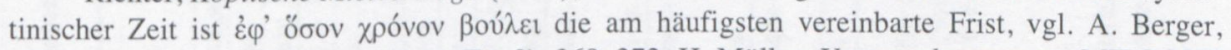

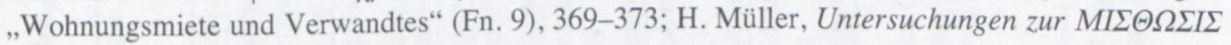
(Fn. 9), 187-192.

18 Vgl. Richter, Koptische Mietverträge (Fn. 1), 143-145.

19 So schon Boud'hors, L'art copte en Égypte (Fn. 2), 201: „L'écriture est assez excercée, presque professionnelle". 


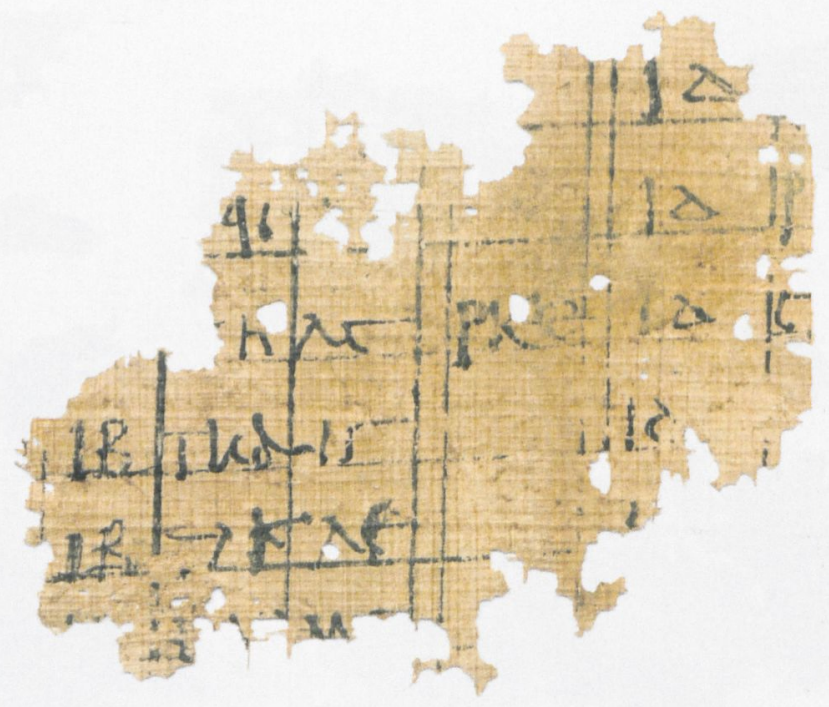

Greek Astronomical Table (P.Carlsb. 726 verso); zu: A. Jones und M. Perale, S. 308ff.

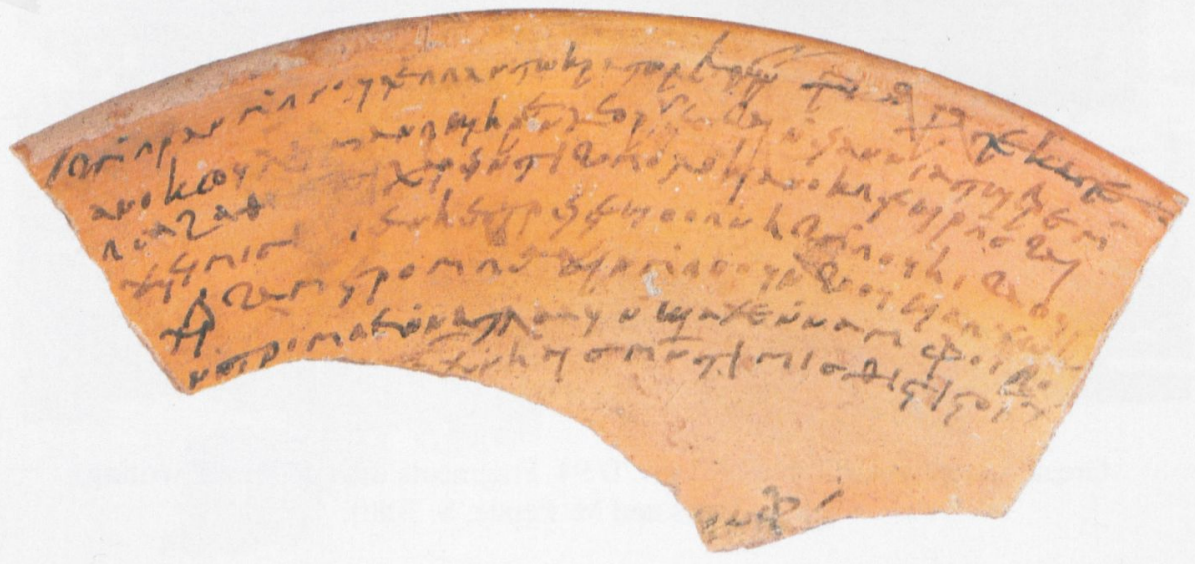

Koptische Mieturkunde (O.Louvre AF 12678); zu: T.S. Richter, S. $360 f f$. 